\title{
Qualidade de Softwares Educacionais Baseados na Web (Semântica): Um Mapeamento Sistemático
}

\author{
Aparecida M. Zem-Lopes (cida.zem@gmail.com) \\ Laís Z. Pedro (laiszagatti@gmail.com) \\ Seiji Isotani (sisotani@icmc.usp.br) \\ Instituto de Ciências Matemáticas e de Computação - ICMC \\ Universidade de São Paulo - USP \\ São Carlos, Brasil
}

\begin{abstract}
Resumo. Softwares educacionais web têm evoluído nos últimos anos, impulsionados pelos avanços das TIC e pela popularização das tecnologias da Web Semântica. Contudo, até o momento são poucas as pesquisas realizadas com o objetivo de desenvolver métodos, técnicas e ferramentas que permitam avaliar a qualidade de softwares educacionais baseados em Web Semântica (SEWS), acompanhando o aumento da demanda e oferta de tais produtos. Assim, o objetivo deste trabalho foi conduzir um Mapeamento Sistemático com o propósito de identificar as pesquisas existentes para avaliação da qualidade de softwares educacionais web. A busca por artigos foi realizada em bases na área de Computação e Educação, sendo 603 artigos selecionados inicialmente. Após leitura e aplicação dos critérios de inclusão e exclusão estabelecidos restaram 78 artigos, lidos integralmente. Os resultados das análises realizadas fornecem um panorama geral sobre os métodos utilizados para avaliação de qualidade dos softwares educacionais web e destacam a precariedade e necessidade de estabelecer fatores e critérios para avaliar a qualidade de SEWS.
\end{abstract}

Palavras-chave: softwares educacionais, web semântica, avaliação de qualidade.

\section{Quality of Semantic Web-based Educational Software: A Systematic Mapping}

Abstract. Web educational software has evolved lately, driven by the advancement of TICs and the popularization of the Semantic Web technologies. Nevertheless, few researches have been conducted to develop techniques and tools to support quality evaluation of semantic web-based educational software. However, identify and establish factors and criteria for assessing the quality of such software is not simple, as it involves, besides those considerations related to any software, the specific to education and Semantic Web. Thus, The aim of this study was conduct a Systematic Mapping for the purpose of identifying methods, models, frameworks and techniques existing for quality assessment of web educational software to establish appropriate approaches to the SEWS from them. The search for articles was conducted in the most relevant electronic databases in the area of Computing and Education, where 603 articles were selected. After reading and applying the criteria, 78 articles remaining. The results of the analyzes provide an overview of methods used for evaluating the quality of web educational software and highlight the need to establish criteria and factors for SEWS.

Keywords: educational software, semantic web, quality assessment. 


\section{Introdução}

A evolução das Tecnologias da Informação e Comunicação (TIC) tem promovido mudanças na maneira como produtos e serviços dos mais diversos setores são projetados, desenvolvidos e disponibilizados no mercado mundial. Um dos setores que se beneficia dessa evolução é o de desenvolvimento de software, impulsionado pela crescente demanda por aplicações cada vez mais eficazes e eficientes. De acordo com Brito et al. (2013), no campo da educação verificam-se melhorias no processo de ensinoaprendizagem motivadas pelo avanço das TIC, as quais facilitam a incorporação de ferramentas educacionais às atividades desenvolvidas pelos professores, por meio da criação de novas possibilidades de ensino, que visam melhorar o aprendizado por parte dos alunos. O software educacional é um exemplo destas ferramentas e pode ser compreendido como um instrumento didático que visa facilitar o processo de ensino e aprendizagem tanto em sala de aula tradicional como no ensino a distância (GARCIA e GARCIA, 2007).

Outro fator que contribui para a evolução dos ambientes e softwares educacionais web é a popularização das tecnologias e aplicativos da Web Semântica. Diversos pesquisadores indicam que a próxima geração de sistemas de apoio à aprendizagem devem possuir algumas características importantes para atender às necessidades dos alunos e professores do século XXI como, por exemplo, ambiente mais adaptável e personalizado, apoio adequado à utilização de pedagogias que melhorem o processo de ensino e aprendizagem, compartilhamento e reuso de informação, novas formas de colaboração entre os alunos, e etc. (BITTENCOURT et al., 2007; 2008).

A Web Semântica pode ser definida como uma nova geração de tecnologias que visa à representação da informação de forma que agentes computacionais consigam interpretá-la. Na Web, em seu formato atual, a informação disponível é representada geralmente em linguagem natural. Essa forma de representação permite que a informação seja compreendida por pessoas, porém, impossibilita sua recuperação e compreensão por computadores ou agentes de software. Para que isso ocorra é necessária uma representação formal e explícita desta informação (ISOTANI et al., 2009). Assim, uma das tecnologias desenvolvidas no âmbito de pesquisa na área de Web Semântica são as ontologias. Uma ontologia pode ser definida como uma representação formal (legível para os computadores) e explícita de uma conceitualização (modelo abstrato de algum fenômeno do mundo real) compartilhada (conhecimento consensual) (BREITMAN, 2005). Por meio delas é possível utilizar os computadores para auxiliar (e automatizar) diversas atividades. Por exemplo, na educação a Web Semântica e as ontologias têm se destacado na solução de problemas de compartilhamento e reuso de material didático, na busca automática de objetos de aprendizagem, e na construção de conteúdos de forma colaborativa (ISOTANI et al., 2009).

Nesse contexto, é também importante que se desenvolva e estabeleça formas e métodos para avaliar a qualidade desses ambientes e/ou softwares educacionais baseados em Web Semântica. No entanto, identificar os requisitos de qualidade de software não é uma tarefa simples. De acordo com Campos e Campos (2001) é preciso considerar as características e tecnologias relativas ao domínio em questão para avaliar um software educacional, além dos critérios referentes à qualidade de software em geral. Pinto Andres e Cybis (2000) discutem sobre as diversas técnicas que podem ser utilizadas para avaliação. As autoras apresentam a dificuldade em se classificar os softwares educacionais quanto a sua qualidade e eficácia, pois tal atividade envolve a interação humana. De acordo com Silva (1999), a qualidade, se classificada em termos de 
ergonomia e pedagogia, extrapola os métodos de avaliação tradicionais, pois para ser avaliada precisa do estabelecimento de critérios de usabilidade (facilidade de uso) além de outros como psicopedagógicos e didáticos.

Nesse contexto, com a finalidade de investigar e compreender o estado da arte da "avaliação de qualidade de softwares educacionais", assim como identificar os trabalhos relacionados a essa pesquisa, um Mapeamento Sistemático da Literatura (MS) foi planejado e executado. Esse MS teve por objetivo identificar modelos, métodos, guidelines, frameworks, técnicas e abordagens desenvolvidas para avaliação de qualidade de softwares educacionais, principalmente aqueles baseados na Web, de forma que se obtivesse um panorama geral sobre o referido tema.

Este trabalho encontra-se estruturado em duas seções, além desta (introdução). A seção 2 apresenta o mapeamento sistemático realizado, dividida em planejamento, condução e relatório. $\mathrm{Na}$ seção 3 são realizadas as considerações finais e, por fim, apresentam-se as referências utilizadas na elaboração deste trabalho.

\section{Mapeamento Sistemático}

A revisão sistemática é uma metodologia adequada para realizar revisão bibliográfica da literatura de forma organizada e sistemática que permite sua condução por meio de etapas bem definidas e proporciona maior confiabilidade e base teórica sólida (KITCHENHAM, 2007). O MS possibilita uma visão mais ampla dos estudos primários para identificar as evidências disponíveis numa determinada área. De acordo com Petersen (2008) o MS fornece uma visão geral da área de pesquisa, possibilitando que se conheça a frequência, quantidade e tipos de estudos referentes às publicações ao longo do tempo, permitindo identificar tendências de pesquisa.

Assim, este trabalho seguiu o processo proposto por Kitchenham (2007) com três fases principais: Planejamento, Condução e Relatório.

\subsection{Planejamento do Mapeamento Sistemático}

$\mathrm{Na}$ fase de planejamento, a partir do estabelecimento do objetivo da pesquisa, deu-se início ao desenvolvimento do protocolo (regras), utilizado para orientar a coleta de evidências do MS. Todas as etapas do MS foram realizadas com o apoio da ferramenta Start ${ }^{1}$.

\section{a) Objetivo}

O MS realizado teve como objetivo identificar modelos, métodos, guidelines, frameworks, técnicas, abordagens desenvolvidas para avaliação de qualidade de softwares educacionais, com foco principalmente em ambientes Web de aprendizagem (também conhecidos como ambientes virtuais de aprendizagem). Com base neste objetivo, definiu-se a seguinte questão de pesquisa:

1. Há algum modelo, método, framework, guideline para avaliação de qualidade de softwares educacionais web?

a. Dentro deste contexto, existe algum método ou modelo de avaliação específico para SEWS?

A partir destas questões, foi possível especificar o PICO (Population, Intervation, Comparison, Outcome), ou seja, população, intervenção, comparação e resultados da seguinte forma:

- População: softwares educacionais web;

- Intervenção (o que será observado): contexto de aplicação e metodologia utilizada nos modelos, métodos, frameworks, guidelines, abordagens,

\footnotetext{
${ }^{1}$ Start - Disponível em: http://lapes.dc.ufscar.br/tools/start_tool
} 
processos, técnicas, métricas, checklists, critérios;

- Comparação: i) em frameworks/guidelines: comparar número passos, duração/tempo para implementação, facilidade de integração; ii) em modelos, técnicas, métodos, processos: facilidade de uso, simplicidade do código, portabilidade.

- Resultados: identificar os modelos, métodos, frameworks, guidelines, técnicas, processos, abordagens mais utilizadas em avaliação de qualidade softwares educacionais web, vislumbrando a possibilidade de extensão para SEWS.

\section{b) Critérios de Inclusão e Exclusão}

De acordo com o protocolo definido para esse MS, foram estabelecidos os critérios de inclusão e exclusão para garantir a seleção de estudos relevantes que pudessem responder às questões de pesquisa, de acordo com o Quadro 1:

Quadro 1. Critérios de inclusão e exclusão

\begin{tabular}{|l|l|}
\hline \multicolumn{1}{|c|}{ INCLUSÃO } & \multicolumn{1}{|c|}{ EXCLUSÃo } \\
\hline $\begin{array}{l}\text { CI-1: Apresenta um framework para } \\
\text { avaliação de softwares educacionais; }\end{array}$ & $\begin{array}{l}\text { CE-1: Estudos que não estejam na língua } \\
\text { portuguesa ou inglesa; }\end{array}$ \\
\hline $\begin{array}{l}\text { CI-2: Documentos que abordem aspectos } \\
\text { práticos de um modelo, método, técnica, } \\
\text { processo, métricas para avaliação de } \\
\text { softwares educacionais; }\end{array}$ & $\begin{array}{l}\text { CE-2: Relatórios, documentos que disponíveis na } \\
\text { forma de resumos ou apresentações ou revisões de } \\
\text { literatura (i.e., revisões e mapeamentos } \\
\text { sistemáticos da literatura); }\end{array}$ \\
\hline $\begin{array}{l}\text { CI-3: Apresenta técnicas bem definidas de } \\
\text { avaliação de softwares educacionais; }\end{array}$ & $\begin{array}{l}\text { CE-3: Estudos que não possuam versões completas } \\
\text { disponíveis; }\end{array}$ \\
\hline $\begin{array}{l}\text { CI-4: Apresenta um método de avaliação } \\
\text { de qualidade de softwares educacionais; }\end{array}$ & $\begin{array}{l}\text { CE-4: Estudos que abordam apenas aspectos } \\
\text { teóricos e filosóficos (sem definição de método, } \\
\text { modelo etc.); }\end{array}$ \\
\hline $\begin{array}{l}\text { CI-5: Apresenta um modelo de avaliação } \\
\text { de softwares educacionais. }\end{array}$ & $\begin{array}{l}\text { CE-5: Estudos que não apresentam relação com } \\
\text { softwares educacionais ou softwares educacionais } \\
\text { web; }\end{array}$ \\
\hline CE-6: Estudos que não abordam avaliação de \\
qualidade de software educacional web;
\end{tabular}

\section{c) Seleção das Fontes de Busca}

A escolha das bases eletrônicas foi baseada em Dieste et al. (2009), que estabelece alguns critérios como a disponibilidade dos estudos primários, cobertura das publicações e conferências relevantes na área de pesquisa, e a busca por estudos no idioma inglês (adotada nos principais eventos e periódicos científicos) e em português, para tentar recuperar estudos importantes no Brasil. Desta forma, as bases consultadas foram: IEEE Xplore, ACM Digital Lybrary, Science Direct (Elsevier), Scopus e Springer. Foram realizadas ainda algumas buscas pontuais, baseadas no grupo de controle (estudos base para o contexto da pesquisa) definido por especialista na área, em conferências específicas.

\section{d) Construção da String de Busca}

A string de busca foi construída de acordo com Kitchenham (2006), por meio da identificação das principais palavras-chave derivadas das questões de pesquisa. Utilizouse os operadores OR e AND, respectivamente para termos sinônimos e termos alternativos de cada palavra chave, definidos como "Model", "Quality evaluation" e "Educational software". Obteve-se como resultado a seguinte string de busca genérica: 
(Model OR Framework OR Guideline OR Process OR Methodology OR Approach OR Metrics OR Checklist OR Criteria) AND ("Quality Evaluation" OR "Quality Evaluating" OR "Quality analysis" OR "Quality validation" OR "Quality Assessment" OR "Evaluate Quality" OR "Assessing Quality" OR "Quality Rating") AND ("educational software" OR "intelligent tutoring system" OR "educational system" OR "educational technologies" OR "educational technology" OR "CSCL" OR "CAI" OR "intelligent virtual environment" $O R$ "virtual learning" $O R$ "educational environment" $O R$ "educational tool" OR "computer aided instruction" OR "computer assisted learning" OR "Learning Management System")

\subsection{Condução do Mapeamento Sistemático}

O processo de condução do MS foi realizado com o apoio das ferramentas Start e Mendeley $^{2}$ no período de outubro/2013 a março/2014 e pode ser resumido na Figura 1.

\begin{tabular}{|l|l|}
\hline 1 & $\begin{array}{l}\text { Primeira etapa (Seleção Inicial) } \\
\text {-Leitura do título, resumo e palavras-chave }\end{array}$ \\
\hline 3 & - Aplicação dos critérios de Inclusão e Exclusão \\
\hline & $\begin{array}{l}\text { Segunda etapa da seleção } \\
\text {-Leitura completa dos estudos }\end{array}$ \\
\hline & - Reaplicação dos critérios de Inclusão e Exclusão \\
\hline & - Terceira etapa (Extração dos dados) \\
\hline & - Análise de qualidade dos dados \\
\hline
\end{tabular}

Figura 1. Resumo do processo de condução do MS

Após a fase de levantamento nas bases de dados foram encontrados 603 estudos no escopo de modelos de avaliação de software educacional.

$\mathrm{Na}$ segunda etapa foram lidos os títulos, palavras-chave e resumos dos trabalhos restando 241, dos quais 67 eram duplicados, o que resultou em 174 estudos. Na terceira etapa, a partir da leitura da introdução e conclusão dos 174 estudos, foram selecionados 78 estudos. A partir desses estudos, foi realizada a leitura completa, para extração das informações de acordo com o protocolo de pesquisa estabelecido. As buscas e seleção dos estudos, para cada base, foi sumarizado no Quadro 2.

Quadro 2. Resumo da condução do MS

\begin{tabular}{|c|c|c|c|c|c|c|}
\hline \multirow[b]{2}{*}{ Base eletrônica } & \multirow{2}{*}{$\begin{array}{l}\text { Busca } \\
\text { inicial }\end{array}$} & \multicolumn{3}{|c|}{$1^{a}$ seleção } & \multicolumn{2}{|c|}{$2^{\mathrm{a}}$ seleção } \\
\hline & & Incluídos & $\begin{array}{l}\text { Excluído } \\
\text { s }\end{array}$ & $\begin{array}{c}\text { Duplicado } \\
\text { s }\end{array}$ & Incluídos & Excluídos \\
\hline IEEE Xplore & $\begin{array}{c}239 \\
(40 \%)\end{array}$ & 94 & 145 & 1 & 36 & 57 \\
\hline ACM Digital Lybrary & $15(3 \%)$ & 13 & 0 & 2 & 7 & 6 \\
\hline SPRINGER & $45(7 \%)$ & 17 & 27 & 1 & 9 & 8 \\
\hline SCIENCE DIRECT & $26(4 \%)$ & 9 & 17 & 0 & 5 & 4 \\
\hline SCOPUS & $\begin{array}{c}273 \\
(45 \%)\end{array}$ & 37 & 173 & 62 & 16 & 22 \\
\hline AD HOC & $5(1 \%)$ & 4 & 0 & 1 & 3 & 1 \\
\hline Total & 603 & 174 & 362 & 67 & 78 & 96 \\
\hline
\end{tabular}

${ }^{2}$ Mendeley - <http://www.mendeley.com/> 


\subsection{Relatório (Sumarização) do Mapeamento Sistemático}

De forma sintética, essa seção apresenta os resultados obtidos no MS realizado, a partir dos estudos primários selecionados, e uma breve análise e discussão desses estudos.

A quantidade de estudos recuperados por ano de publicação aumentou principalmente a partir do ano 2009 e em 2012 houve a maior incidência de estudos, confirmando a crescente preocupação da comunidade científica com a qualidade dos softwares educacionais web (Figura 2).

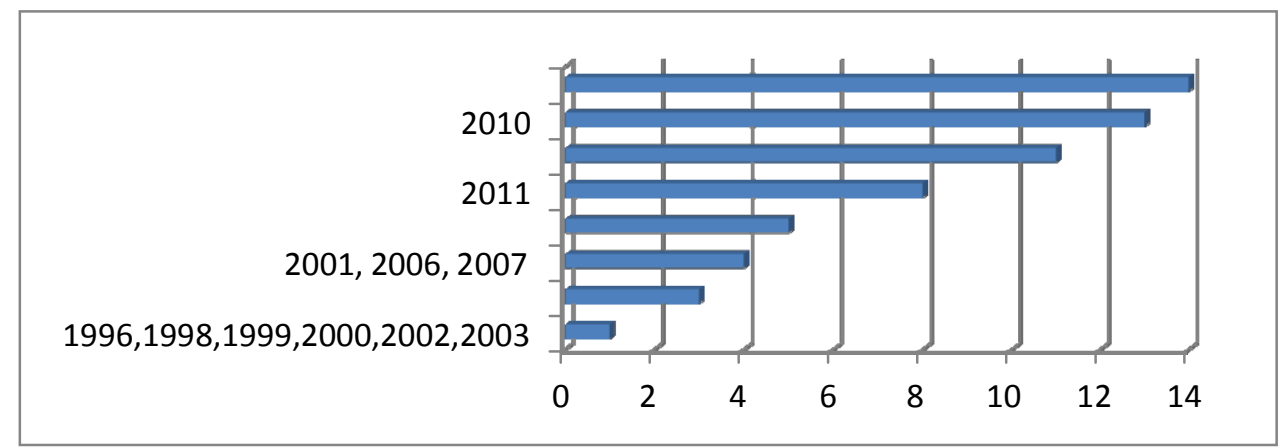

Figura 2. Estudos relacionados a qualidade de software por ano de publicação

Com relação ao país de origem dos autores, verificou-se que China, Inglaterra, Brasil e Espanha são os mais ativos no tema de avaliação de software educacional web. A Figura 3, mostra a nuvem de palavras com os países dos autores e a respectiva quantidade de publicações. Quanto maior a quantidade de publicações maior é o tamanho da fonte utilizada.

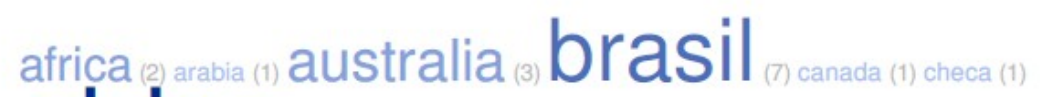 china esloveniaa) espanha grecia $_{(\text {mati }}$ inglaterra

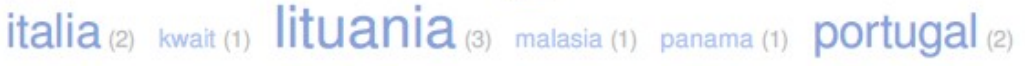 republica (1) romenia (2) saudita (1) SUl (3) taiwan (1) turquia (2)}

Figura 3. Publicações por país

Para responder a questão de pesquisa principal do trabalho (i.e. há algum modelo, método, framework, guideline para avaliação de qualidade de softwares educacionais $w e b ?$ ) analisou-se profundamente cada um dos 78 artigos selecionados. Como um dos resultados, verificou-se que $82 \%$ deles (51 artigos) apresentam meios de avaliação de software educacional web. Dentre eles, nenhum é específico para avaliar softwares educacionais baseados em Web Semântica, respondendo assim, a questão de pesquisa secundária do trabalho (i.e. dentro deste contexto, existe algum método ou modelo de avaliação específico para SEWS?).

Para discutir um pouco mais as contribuições dos artigos selecionados no mapeamento, será feita uma separação dos critérios, fatores e requisitos necessários para avaliar softwares educacionais de acordo com Varlamis e Apostolakis (2010), que identificam quatro aspectos importantes na avaliação de qualidade: pedagógicos, técnicos, sociais e organizacionais. 
Os aspectos pedagógicos levam em consideração a qualidade do processo de aprendizagem e está fortemente relacionado com a obtenção dos objetivos de ensino e aprendizagem desejados pela tripla aluno-professor-instituição. Dentre os artigos analisados, 37\% (i.e. 29 estudos primários) utilizaram ou propuseram aspectos pedagógicos para avaliar a qualidade de um software educacional Web. Por exemplo, os trabalhos de Squires e Preece (1996), Ardito et al. (2006), Spyropoulou et al. (2013) fazem adaptações de técnicas e fatores relacionados a usabilidade para propor meios de avaliar a qualidade dos softwares educacionais web tanto do ponto de vista de interação do aluno com os diversos agentes/objetos do ambiente (interface do software web, professor, material, conteúdo, outros alunos, etc) quanto do ponto de vista teóricodidático (eg. Behaviorismo, Construtivismo, Cognitivismo, etc) que rege os processos utilizados para ensinar ou auxiliar o aluno a construir o seu próprio conhecimento. Um outro exemplo é a abordagem proposta por Yueshun e Ping (2009) que faz uso de mineração de textos para identificar as percepções, atitudes e falhas dos alunos no ambiente e assim propor um método para avaliação de qualidade.

Os aspectos técnicos estão relacionados com a infraestrutura e funcionalidades que viabilizam o aprendizado por meio de softwares educacionais web. Neste contexto, 34\% (i.e. 27 estudos primários) investigaram aspectos técnicos no contexto de avaliação de softwares educacionais web. Dentre eles, podemos destacar a reusabilidade de objetos de aprendizagem como um dos importantes critérios para avaliação de qualidade, pois aumenta a vida útil do material didático criado e a sua posterior utilização em diferentes situações e contextos (Sanz-Rodriguez et al., 2010). Um outro aspecto importante citado por diversos autores é a flexibilidade e adaptabilidade do software para atender tanto um grupo diversificado de usuários quanto para lidar com diferentes tipos de materiais didáticos e metodologias de ensino-aprendizagem sem perder a consistência da interface de interação entre o usuário e o software (Lanzilotti et al., 2006; Kurilovas e Dagien, 2009; Celik, 2012). Outros critérios como segurança, privacidade e velocidade (do inglês responsiveness) também são aspectos técnicos importantes identificados pela comunidade (Silius et al., 2011; Wang et al., 2012).

Os aspectos sociais avaliam o potencial que o software oferece para que os seus usuários possam interagir entre si, avaliar a comunidade e seus membros, ser acessível para pessoas com necessidades especiais, viabilizar a criação de comunidades de prática, além de auxiliar na criação e condução de atividades de aprendizagem colaborativa. Atualmente, a qualidade do software está intrinsicamente relacionada funcionalidades de colaboração e comunicação devido as necessidades dos usuários em trabalhar de maneira integrada dentro do ambiente. Dentro os trabalhos analisados neste mapeamento 11\% (i.e.

9 estudos primários) apresentaram alguns critérios de avaliação de qualidade abordados aspectos sociais. Um dos aspectos que teve grande destaque entre os estudos analisados foi o de acessibilidade. Segundo, Martin et al. (2007) e Nanduri et al. (2012) frequentemente os alunos com alguma necessidade especial encontram mais dificuldade de interação no ambiente virtual do que no presencial. Isso ocorre, pois a interação no ambiente virtual está baseada em diversas mídias e meios de comunicação, e poucas são aquelas adaptadas para lidar com usuários com alguma necessidade especial. Dessa forma, para aumentar a inclusão dos usuários este fator torna-se um importante critério para avaliar a qualidade de um software educacional.

Finalmente, os aspectos organizacionais fazem referência a eficiência e custo para gerenciar as diversas "peças" que envolvem o meio educacional como os atores (alunos, professores, gestores, pais, etc), tecnologias (computadores, tablets, softwares, etc), processos e ambientes. Um software educacional web pode auxiliar a gerenciar o meio educacional ou ser apenas mais uma "peça" que precisa ser adequadamente 
encaixada. A efetividade do uso do software educacional seja por alunos quanto por professores é fortemente dependente dos aspectos organizacionais e, portanto, a avaliação da qualidade do software deve levar em consideração este contexto organizacional. Neste mapeamento, 15\% dos artigos (i.e. 12 estudos primários) investigaram a avaliação de softwares educacionais web sob esta perspectiva. Por exemplo, o trabalho de Militaru et al. (2012) apresentam um framework, a partir de uma versão modificada do framework SEEQUEL (Core Quality Framework), usado para construir a árvore de qualidade a partir de características comuns extraídas da experiência com vários ambientes de e-learning. Dentre eles, estão aspectos organizacionais relacionados com os recursos humanos e as qualidades técnicas necessárias para gerenciar as atividades de ensino. Outro trabalho que enfatiza alguns dos elementos organizacionais necessários para avaliar a qualidade do software é apresentado por Al-Sharhan et al., (2012)

Além dos fatores apresentados, alguns outros como correção, integridade, flexibilidade, testabilidade, interoperabilidade, capacidade para monitorar o projeto aprendizagem, interação aluno e ambiente, interação aluno e aluno, conteúdo confiável e atualizado, qualidade técnica e estética compõem estes grupos de critérios e estão presentes em vários estudos do MS para criação de abordagens em frameworks de avaliação (KELLY, 2008; WANG; CHENG; ZHENG, 2010).

Kelly (2008) apresenta um processo colaborativo para avaliar, guiar e selecionar novas e emergentes tecnologias educacionais e promove uma discussão sobre como um processo de avaliação desse tipo pode abranger stakeholders interdisciplinares, a partir destes fatores e critérios mencionados. Além disso, vislumbra a aplicação dessas tecnologias em contextos reais de ensino e aprendizagem, integrando as disciplinas. A metodologia utilizada involve o design e a identificação de novas atividades de aprendizado, o desenvolvimento de critérios no qual mapeia os objetivos das atividades de aprendizagem e a incorporação de tecnologias que permitam a realização de atividades dentro do curso.

Já a abordagem discutida por Sanz-Rodriguez et al. (2010) objetiva ajudar na seleção de materiais educativos reutilizáveis a partir de repositórios na Web e desenvolver um indicador da capacidade de reutilização dos objetos de aprendizagem.

Outros trabalhos identificados na literatura contribuem para facilitar a complexa tarefa de avaliação de qualidade, como o de Nanduri et al. (2012) que propuseram um framework para avaliação de qualidade de ambientes e-learning e dos conteúdos desses, com base nas normas vigentes e melhores práticas relacionadas aos parâmetros de qualidade para essas aplicações. Conformidade com os padrões, satisfação do usuário e avaliação programática são os principais fatores abordados por Nanduri et al. (2010) ao projetar esse framework.

\section{Considerações Finais}

A evolução das TIC nos últimos anos e consequente avanço das tecnologias da Web Semântica, aliados à demanda crescente por ambientes e softwares educacionais que permitam o compartilhamento, interoperabilidade, personalização, reuso etc. dos conteúdos educacionais, motivam cada vez mais o desenvolvimento de ambientes e SEWS. A partir do MS da Literatura realizado pode-se observar que existem diversas abordagens para avaliação de softwares educacionais web. Porém, nos estudos analisados não foi possível identificar nenhuma abordagem que trate da avaliação de SEWS. Nesse contexto, o MS permitiu confirmar demanda por uma abordagem que consiga dar conta de avaliar os referidos softwares apontando para uma lacuna na literatura, que pode ser explorada num futuro próximo, para contribuir com profissionais, desenvolvedores e 
outros usuários (professores, educadores, alunos, mediadores, tutores) que desejam ou necessitam adquirir e utilizar um SEWS.

\section{Agradecimentos}

Os autores agradecem o CNPq e a FAPESP pelo apoio financeiro concedido.

\section{Referências}

AL-SHARHAN, S.; AL-HUNAIYYAN, A. Towards an effective integrated e-learning system: Implementation, quality assurance and competency models. In Seventh International Conference on Digital Information Management, p.274-279, 2012.

ARDITO, C.; COSTABILE, M. F.; DE MARSICO, M.; LANZILOTTI, R.; LEVIALDI, S.; ROSELLI, T.; ROSSANO, V. An approach to usability evaluation of e-learning applications. Universal Access in the Information Society, v. 4, n. 3, p. 270-283, 2006.

BITTENCOURT, I. I.; DE BARROS COSTA, E.; ALMEIDA, H. O. de; FONSECA, B.; MAIA, G.; CALADO, I.; SILVA, A. P. da. Towards an ontology-based framework for building multiagent intelligent tutoring systems. In: Simpósio Brasileiro de Engenharia de Software. Workshop on Software Engineering for Agent-oriented Systems, 2007.

BITTENCOURT, I. I.; ISOTANI, S.; COSTA, E.; MIZOGUCHI, R. Research Directions on Semantic Web and Education. Journal Scientia, v. 19, n. 1, p. 59-66, 2008.

BREITMAN, K.Web Semântica: a internet do futuro. Rio de Janeiro: LTC, 2005.

BRITO, L. M. DE; GIUBERTI JÚNIOR, J. R.; GOMES, S. G. S.; MOTA, J. B. Ambientes virtuais de aprendizagem como ferramentas de apoio em cursos presenciais e a distância. Revista Novas Tecnologias na Educação, v. 11, n. 1, julho, 2013.

CAMPOS, G. H. B.; CAMPOS, F. C. A. Qualidade de aplicações hipermídia. Capítulo publicado no livro Qualidade de software: Teoria e Prática. Campinas: Makron, 2001.

CELIK, S. (2012) Development of usability criteria for e-learning content development software. Turkish Online Journal of Distance Education, v.13, n. 2, p. 336-345, 2012.

DIESTE, O.; GRIMÁN, A.; JURISTO, N. Developing search strategies for detecting relevant experiments. Empirical Software Engineering, v. 14, n. 5, p. 513-539, 2009.

GARCIA, I.; GARCIA, J. An alternative methodology to develop educational software based on effective practices. The Second International Conference on Systems and Networks Communications ICSNC 2007, Cap. Esterel, French Riviera, France, 2007.

ISOTANI, S.; BITTENCOURT, I. I.; MIZOGUCHI, R.; COSTA, E. Estado da arte em web semântica e web 2.0: potencialidades e tendências da nova geração de ambientes de ensino na internet. Revista brasileira de informática na educação, v. 17, n. 1, 2009.

JACKO, J. A. AND STEPHANIDIS, C. Human-computer Interaction: Theory and Practice. Part I. Lawrence Erlbaum Associates, Incorporated. 2003.

KELLY, G. A collaborative process for evaluating new educational technologies. CampusWide Information Systems, v. 25, n. 2, p. 105-113, 2008.

KITCHENHAM, B. Procedures for performing systematic reviews. Keele, UK, Keele University, 2004. Disponível em: http://testszingarelli.googlecode.com/svnhistory /r336/trunk/2-Disciplinas/MetodPesquisa/kitchenham_2004.pdf>. Acesso em $01 \mathrm{dez} .2013$.

KITCHENHAM, B. Empirical paradigm - the role of experiments. Empirical software engineering issues: critical assessment and future directions, Heidelberg. Springer-Verlag, 2006.

KURILOVAS, E.; DAGIENĖ, V. Multiple Criteria Comparative Evaluation of E-Learning Systems and Components. Informatica, v. 20, n. 4, p. 499-518. 2009. 
LANZILOTTI, R., ARDiTO, C., \& COSTABILE, M. F., \& DE ANGELI, A. eLSE Methodology: a Systematic Approach to the e-Learning Systems Evaluation. Educational Technology \& Society, v. 9, n. 4, p. 42-53, 2006.

MILITARU, T.; SUCIU, G.; TODORAN, G. The evaluation of the e-learning applications' quality. ELMAR, 2012 Proceedings, (September), p.12-14, 2012.

NANDURI, S.; BABU, N. S. C.; JAIN, S.; SHARMA, V.; GARG, V.; RAJSHEKAR, A. P.; RANG, V. Quality Analytics Framework for E-learning Application Environment. IEEE Fourth International Conference on Technology for Education, p. 204-207, 2012.

PETERSEN, K.; FELDT, R. Systematic mapping studies in software engineering. ... in Software Engineering, p.1-10, 2008. Disponível em: <http://robertfeldt.net/ publications/petersen_ease08_sysmap_studies_in_se.pdf >. Acesso em 01 dez. 2013.

PINTO ANDRES, D.; CYBIS, W. de A. Um Estudo Teórico sobre as Técnicas de Avaliação de Software Educacional. VI Congreso Argentino de Ciencias de la Computación, 11 p., 2000.

ROQUE, G. O. B.; FONSECA, M. V. de A.; CAMPOS, G. H. B. de. A segunda geração da avaliação da qualidade de cursos a distância e os atributos do saber fazer. B. Téc. Senac: a R. Educ. Prof., Rio de Janeiro, v. 38, n. 2, maio/agosto, 2012.

SANZ-RODRIGUEZ, J.; DODERO, J. M.; SANCHEZ-ALONSO, S. Metrics-based evaluation of learning object reusability. Software Quality Journal, v. 19, n. 1, p.121-140, 2010

SILIUS, K.; KAILANTO, M.; TERVAKARI, A. Evaluating the Quality of Social Media in an Educational Context. International Journal of Emerging Technologies in Learning, v. 6, n. 3, p. 21-27, 2011.

SILVA, C. R. Avaliação da Qualidade de Software Educacional. In: Encontro Nacional de Engenharia de Produção. Anais... Rio de Janeiro, 1999.

SHNEIDERMAN, B.; BEN, S. Designing the user interface: strategies for effective humancomputer interaction. 4/e (New Edition). Pearson Education India. 2003.

SQUIRES, D.; PREECE, J. Usability and learning: evaluating the potential of educational software. Computers \& Eduction, v. 27, n. 1, p. 15-22, 1996.

SPYROPOULOU, M.; NTOUROU, D.; SIMAKI, V.; MALAGKONIARI, D.; KOUMPOURI, A.; SORRA, M. Evaluating the correspondence of educational software to learning theories. In Proceedings of the 17th Panhellenic Conference on Informatics. ACM Press p. 250-257, 2013.

VARLAMIS, I.; APOSTOLAKIS, I. A Framework for the Quality Assurance of Blended ELearning Communities. International Conference on Knowledge-Based and Intelligent Information and Engineering Systems. LNCS 6278, p. 23-32, 2010

WANG, Y.; CHENG, Y.; ZHENG, Z. Evaluation design of learners' online learning quality in network learning platform. In International Conference on Artificial Intelligence and Education, p. 222-225, 2010.

WANG; Y.; ZHANG, L.; CAI, H.; SUN, J.; LI, N. Evaluating the Quality of Distance Education Services by Using Modern Information Technology. In IEEE Asia-Pacific Services Computing Conference, pp.192-199, 2012. 